\title{
Extracellular vesicles as therapeutic tools in cardiovascular diseases
}

\section{Audrey Fleury, Maria Carmen Martinez* and Soazig Le Lay*}

INSERM U1063 "Oxidative Stress and Metabolic Pathologies," Institut de Biologie en Santé, Université d'Angers, Angers, France

Edited by:

Francesc E. Borras, Institut

$d^{\prime}$ Investigació en Ciències de la Salut

Germans Trias i Pujol, Spain

\section{Reviewed by:}

Maria Mittelbrunn, National Center of Cardiovascular Research, Spain

Martial Ruat, INSERM, France

\section{*Correspondence:}

Maria Carmen Martinez and Soazig

Le Lay, INSERM UMR 1063

"Oxidative Stress and Metabolic pathologies," Institut de Biologie en

Santé, Université d'Angers, 4 rue

Larrey, Angers F-49933, France

e-mail: carmen.martinez@

univ-angers.fr;

soazig.lelay@inserm.fr
Extracellular vesicles (EVs), including microvesicles (MVs) and exosomes, are small vesicles secreted from a wide variety of cells. Whereas MVs are particles released by the outward budding of the plasma membrane, exosomes are derived from endocytic compartments. Secretion of EVs can be enhanced by specific stimuli, and increased plasma circulating levels of EVs have been correlated with pathophysiological situations. MVs, already present in the blood of healthy individuals, are considerably elevated in several cardiovascular diseases associated with inflammation, suggesting that they can mediate deleterious effects such as endothelial dysfunction or thrombosis. Nonetheless, very recent studies also demonstrate that MVs may act as biological information vectors transferring proteins or genetic material to maintain cell homeostasis, favor cell repair, or even promote angiogenesis. Additionally, exosomes have also been shown to have pro-angiogenic and cardio-protective properties. These beneficial effects, therefore, reveal the potential therapeutical use of EVs in the field of cardiovascular medicine and regenerative therapy. In this review, we will provide an update of cellular processes modulated by EVs of specific interest in the treatment of cardiovascular pathologies. A special focus will be made on the morphogen sonic hedgehog (Shh) associated with $\mathrm{EVs}_{\mathrm{s}}\left(\mathrm{EVS}_{\mathrm{Sh}}^{\mathrm{Sh}}\right)$, which have been shown to mediate many pro-angiogenic effects. In addition to offer a potential source of cardiovascular markers, therapeutical potential of EVs reveal exciting opportunities to deliver specific agents by non-immunogenic means to cardiovascular system.

Keywords: extracellular vesicle, microparticles, microvesicles, exosomes, cardiovascular diseases, angiogenesis, sonic hedgehog, therapeutical tools

\section{INTRODUCTION}

Extracellular vesicles (EVs) comprising exosomes $(<100 \mathrm{~nm}$ of size), microparticles, and apoptotic bodies (100-1200 $\mathrm{nm}$ in size) are physiologically released by almost all cell types and represent endogenous cargos that are able to participate in cell-to-cell communication. The composition of EVs determines the type of message, which they can convey. Indeed, EVs are rich in proteins, mRNAs, and miRNAs as well as lipids, which they can transfer to target cells by ligand/receptor interaction, fusion, and/or internalization. Manipulation of cells generating EVs by pharmacological treatment or transfection allow to produce EVs rich in specific components that can affect selectively the functions of target cells. In this article, we will review the current knowledge of the function and impact of EVs used as therapeutic tools against cardiovascular diseases.

\section{EVs: POTENTIAL DELIVERY TOOLS/VECTORS IN THE CARDIOVASCULAR SYSTEM}

In addition to their different size, the criteria that are used to distinguish between the various types of EVs are based on the cellular compartment that they originate from and on their composition (1). While exosomes are formed from the multivesicular bodies, both microparticles and apoptotic bodies come from plasma membrane. Obviously, composition of EVs depends on the type of (i) cells that they are from and (ii) stimulation that generates their release. Thereby, specific antigens carried by EVs allow the identification of donor/producer cell, whereas use of different agents to stimulate EV generation allows achieving EVs with different potential effects.

Extracellular vesicles have been isolated from the main fluids of the organism and from several solid tissues (tumors, atheroma plaque, skeletal muscle...). However, it is difficult to establish the exact nature of EVs from solid tissues, as the recovered materials can also comprise intracellular vesicles released during the tissue dissociation process (2). In addition to their physiological role during development and homeostasis, some pathophysiological situations will enhance release of EVs and affect composition of EVs or both. Thus, during cardiovascular diseases, both elevated levels and different composition of EVs have been described suggesting that EVs may represent potential novel targets for therapeutic intervention $(3,4)$. Of note, circulating endothelial EVs were associated with the presence of cardiometabolic risk factors in a community cohort underscoring the potential influence of high-risk metabolic profiles on endothelium activation or injury (5). Given the fact that elevated EV levels often correlate with the severity of cardiovascular diseases, one strategy would consist in reducing circulating EVs to normal levels in order to prevent associated deleterious signaling. In this perspective, many ongoing studies are designed to evaluate changes in circulating EV levels in response to pharmacological treatment used [for review, see 
Ref. (6)]. However, such strategies do not allow distinguishing between effects of drugs on EV generation and/or EV clearance. An alternative to modulate EV production would be either to act on processes regulating their formation and/or release or to inhibit their interaction with target cells through specific targeting of their components (receptors, ligands, lipids. ..) (7). Notably, inhibition of ceramide formation by the use of amiloride drug reduces significantly mouse and human tumor cell growth by blocking secretion of heat shock protein-72 (HSP 72) EV-associated protein (8). While no studies using such strategies have been yet described in cardiovascular field, a limiting step is also linked to the lack of specificity of targeting mechanisms regulating EV formation and release, which may produce many unwanted effects.

Alternatively, EVs constitute promising therapeutic delivery tools in cardiovascular diseases. Their general capacity to act as bioactive cargoes, particularly through their ability to carry secretory molecules such as cytokines, chemokines, or growth factors as well as exogenous nucleic acids, highlight them as attractive vehicles (9). In addition, EVs are able to use the vasculature to signal at considerable distance from their donor cell origin. In this respect, central nervous system-derived EVs could enter the bloodstream and communicate with endothelial cells in the peripheral circulation and with cells involved in immune surveillance (10).

Although largely identified as deleterious carriers, EVs might also have beneficial effects and deliver protective messages to preserve endothelial function and/or vascular integrity. Indeed, circulating EVs from sepsis patients are able to restore vascular hyporeactivity in vessels treated by lipopolysaccharide (11). In agreement, high circulating levels of EVs correlate with survival in sepsis patients (12). Besides this EV-mediated adaptative/protective response, EVs have demonstrated regenerative capacities, mainly when they derived from progenitor or mesenchymal stem cells (MSC), therefore, opening perspectives to use their innate properties in regenerative medicine (Figure 1).

\section{THERAPEUTIC USE OF MSC-DERIVED EVs IN CARDIAC REGENERATIVE MEDICINE}

Cardiac regenerative therapy, based on intramyocardial injection of MSCs, has been in the last few years one of the most promising approaches to both prevent cardiac damage and allow
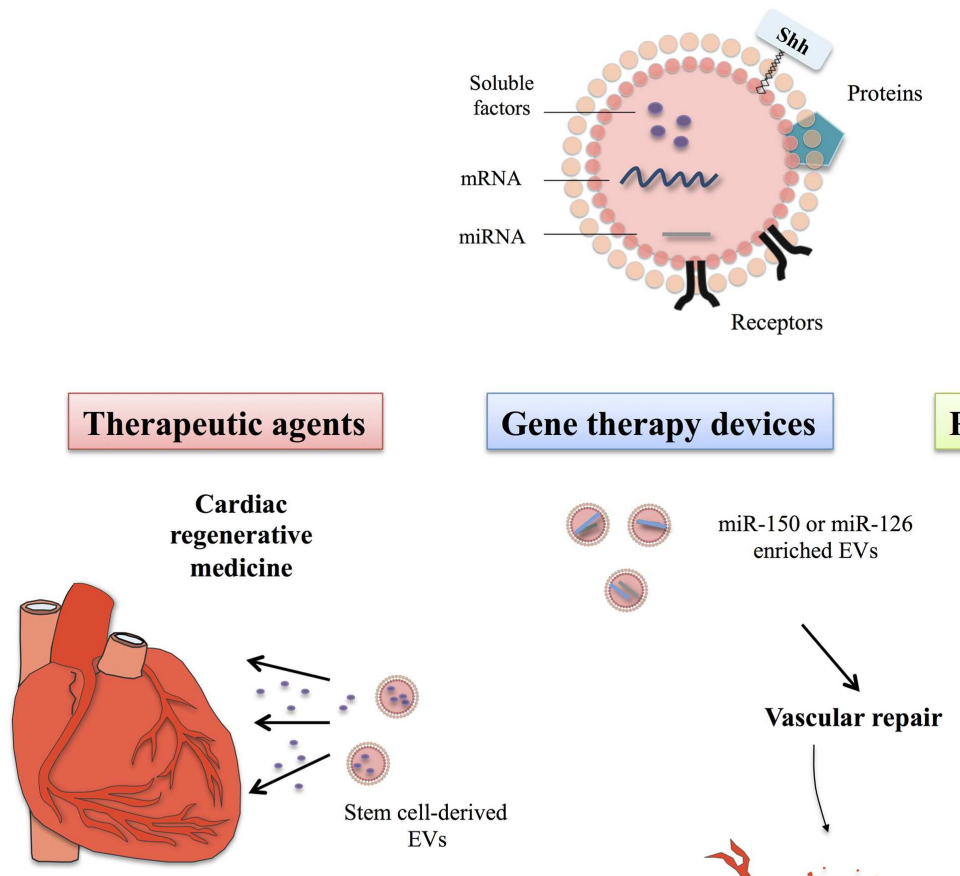

Protein delivery carriers

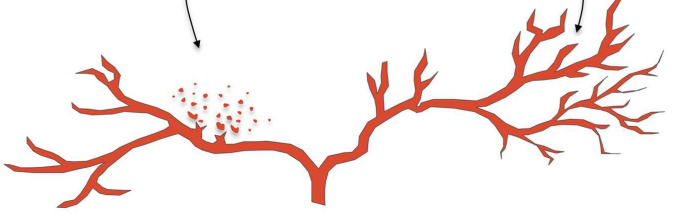

FIGURE 1 |Therapeutical potential of extracellular vesicles in the context of cardiovascular disorders. Extracellular vesicles (EVS) constitute bioactive cargoes, particularly through their ability to carry proteins, receptors but also genetic material including microRNA (miRNA) or mRNA. Since EVs participate actively in the modulation of many physiological processes, they can be used as therapeutic agents in cardiac regenerative medicine. Improvement of cardiac function depends on the EV-transfer of specific factors, such as soluble proteins, growth factors, lipids, or genetic material. Use of stem cell-derived EVs in cardiac regenerative therapies, therefore, allows delivering specific signals by non-immunogenic means to heart. As EVs naturally contain genetic material, cell sources can be genetically manipulated to produce EVs specifically loaded with miRNA of choice, such as miR-150 or miR-126, described as important regulators of angiogenesis or vascular integrity. Treatment of endothelial cells or vascular tissues with these miRNA-enriched EVs has demonstrated their protective and regenerative properties on cardiovascular system. Specific culture system might also allow to specifically targeting molecules of interest to EVs, like the morphogen Shh that is an important regulator of injury-induced angiogenesis and neovascularization. Use of EVs ${ }^{\mathrm{Shh}+}$ even appears in this context more powerful than classical recombinant Shh protein, likely linked to its localization within natural EV membranous environment. 
cardiac repair. However, experimental or clinical studies demonstrate insufficient cardiomyocyte or vascular cell differentiation to account for improvements of cardiac function (13). This led to formulate the paracrine-hypothesis according to which soluble factors derived from MSCs would be responsible for beneficial outcomes $(14,15)$. First evidences establishing that cellular secretions are cardio-protective resulted from administration of MSC-derived conditioned medium to rodents and pigs, which results in reduced infarct size and led to improved heart function (16-18). Also, exosomal fraction contained in MSCconditioned medium mediates preservation of cardiac function after ischemia/reperfusion injury (19). These protective effects likely result from decreased oxidative stress and activation of PI3K/Akt pathway, which enhance myocardial viability and prevent adverse cardiac remodeling (20). Of note, EVs derived from bone marrow $\mathrm{CD}^{+}{ }^{+}$stem cells or endothelial progenitor cells (EPCs) have been shown to promote angiogenesis $(21,22)$.

Of great interest is the recently reported potential of EVs, particularly exosomes, derived from cardiac progenitor cells (CPC). Indeed, CPC-derived EVs have been shown to stimulate migration of endothelial cells (23) and to protect ischemic myocardium from acute ischemia/reperfusion injury (24). This would, therefore, open new perspectives to directly used EVs derived from cells present in the heart itself. Accordingly, electron microscopic imaging of CPCs and adult mouse hearts has revealed the release of vesicles, which would have the morphological characteristics of EVs (25).

Altogether, the use of stem cell-derived EVs instead of stem cells engraftment might open new perspectives for cardiac regenerative therapies. This would first resolve safety concerns related to uncontrolled dissemination of transplanted cells or aberrant stem cell differentiation. In addition, immunosuppressive effects reported for certain EVs could favor efficient cardiac tissue regeneration (26). Moreover, since exosomes could be easily stored and keep their biological properties over an extended storage period, they can overcome many of the limitations linked to the use of viable cells in regenerative medicine. However, further investigations would be needed to optimize their characterization, quality, and purification in order to accurately control their production in the perspective of clinical uses (27). In particular, pro or antitumor effects reported for MSC-derived EVs would need to be fully understood and highly regulated $(28,29)$.

\section{EVs AS GENE-THERAPY DEVICES}

As described above, EV composition and cargoes transported (proteins, genetic material, lipids) vary with cell origin. In addition to use them as delivery vector for specific proteins, their ability to transport and deliver genetic material to non-adjacent cells have highlighted their potential as new delivery vectors in gene-therapy (see Figure 1).

Extracellular vesicles derived from stem cells are reported as carriers for nucleic acids, which by horizontal transfer of mRNAs are able to reprogram hematopoietic progenitors (30). Subsequently, EPCs are also shown to activate angiogenesis in endothelial cells by shuttling specific mRNA-derived EVs associated with the phosphoinositide 3-kinase (PI3K)/AKT signaling pathway, therefore, inducing angiogenic responses (31).
Further investigations have demonstrated that delivery of mRNAs was not restricted to MVs but could be extended to exosomes derived from mast cells (32) and could be translated once after entering another cell (32-34). Furthermore, detailed RNA analysis of total RNAs derived from EVs from various cell types also revealed the presence of small RNAs, including microRNAs (miRNA) $(32,35,36)$. Transfer of miRNAs contained in exosomes derived from dendritic cells operates via fusion of cells, leading to the release of the exosomes content into the dendritic cell cytosol (37).

Numerous clinical studies have revealed that cardiovascular diseases correspond with specific signature patterns of miRNA expression $(38,39)$. Circulating EVs represent transport vehicles for large numbers of specific miRNAs involved in fundamental cellular processes related to cardiovascular disorders (40). Importantly, the comparison of ratio of miRNA expression in circulating EVs to that in EV-free plasma reveals the majority of circulating miRNAs were present in EVs (41). Since miRNA profile in EVs is significantly different from their maternal cells, an active mechanism of selective "packaging" from cells into EVs likely occurs. Of note, molecular packaging of miRNA-loaded exosomes is controlled through the recognition of sequence motifs present in miRNAs by sumoylated protein hnRNPA2B1 (heterogeneous nuclear ribonucleoprotein A2B1) (42). Moreover, this compartmentalization may be regulated by external stimuli as illustrated by hypoxia, which can modulate immunomodulatory and regenerative properties of MSC-derived EVs (16) but also enhanced expression of pro-angiogenic miRNAs in EPC-derived EVs (43).

MiRNAs contained in EVs might efficiently regulate target genes in recipient cells as illustrated with monocyte miRNA150 , selectively packaged into EVs following treatments of human monocyte/macrophage THP1 cells with inflammatory factors (41). By the use of THP-1-derived EVs expressing a mimic miR150 , the authors showed elevated miR-150 levels in in vitro EVtreated endothelial cells as well as in mouse blood vessels following EV intravenous injection. Consequently, protein level of c-Myb, a gene targeted by miR-150, is effectively reduced resulting in enhanced endothelial cell migration. This monocyte/macrophage EVs delivery system has been further optimized by transfecting donor cells with a chemically modified miRNA (miR-143), which revealed higher stability (44).

MiR-126, an important regulator of angiogenesis and vascular integrity $(45,46)$ is enriched in different types of EVs, such as apoptotic bodies derived from endothelial cells (47), endothelial microparticles (48), or even EPCs (49). In agreement with the striking evidence describing the role of miR-126 in the regulation of vascular integrity on the molecular level, treatment with these different types of miR-126-enriched EVs all promote vascular regeneration. Indeed, systemic treatment of hypercholesterolemic $\mathrm{ApoE}^{-/-}$mice with miR-126-enriched EVs limited atherosclerosis and increased $\mathrm{Scal}^{+}$cell incorporation into aortic plaques (47) whereas miR-126-associated EVs promote reendothelialization in vivo (48). Alternatively, both angiogenic miR-126 and miR-296 participate in the improvement of neovascularization observed in a model of hindlimb ischemia induced in SCID mice following treatment with EPC-derived EVs (49). Of particular interest, miRNA profiling in patients with coronary artery disease 
has shown significantly reduced levels of miR-126 in comparison with healthy controls (50) or even total loss circulating miR-126 in patients with diabetes mellitus (39). Therefore, development of EV-based miR-126 therapeutic strategy might be promising in the context of metabolic and cardiovascular diseases.

Our group has developed one type of EVs carrying the morphogen Shh that reveals protective properties on cardiovascular cells. The next part of this review is focused on the description of the effects of these EVs.

\section{EVs HARBORING Shh AS VASCULAR AND CARDIO-PROTECTIVE DELIVERY TOOLS}

\section{IMPORTANCE OF Shh SIGNALING IN VASCULAR FUNCTION}

Shh is a morphogen that belongs to the conserved hedgehog $(\mathrm{Hh})$ protein family. Hedgehog signaling is essential in vertebrates for embryo patterning, as it participates in central nervous system development, lateral asymmetry, and anterior-posterior limb axis (51). In adult tissues, Shh signaling has been implicated in cell proliferation, differentiation, and survival in a wide variety of target tissues/organs [reviewed in detail in Ref. (52)]. Hh proteins are covalently linked to cholesterol at its carboxyl terminus (53) and palmitoylated at its amino terminus (54). Both lipid modifications influence activities of Shh $(55,56)$ and long-range spread of Shh signaling $(57,58)$. Diffusion of dual-lipid modified Shh proteins apparently occurs via secretion as mono or large soluble multimers, further transported via lipoproteins (59) or in EVs (60-62). A major component of the Hh pathway includes the 12 transmembrane receptor PTC (Patched) that constitutively represses Hh signaling. Binding of Shh to PTC inhibits the repression of Smoothened (SMO), which functions as G protein-coupled receptor (GPCR). This derepression leads to the activation of zincfingers transcriptions factors Gli, which translocate to the nucleus where they act as activators or repressors of target gene transcription. Besides this classical-Shh signaling pathway, referred as "canonical pathway," other non-canonical pathways that do not clearly act via the Shh-Smo-Gli axis have been reported, which regulate many cellular processes including cell energy metabolism $(63,64)$.

Shh signaling is crucial in new vessel formation during embryogenesis and organogenesis. Its role in angiogenesis and neovascularization has been previously reviewed in details (65). Hh signaling components are present in adult cardiovascular tissues and can be activated in vivo (66). In adult tissue, activation of Shh signaling, via recombinant Shh or gene-therapy transfer, triggers cellular responses in a wide variety of vascular cells (endothelial, EPCs, smooth muscle cells, fibroblasts) promoting angiogenesis and protecting against ischemic injuries (66-68). These Shh effects on cardiovascular system seem to operate via complex pathways, involving Gli-dependent and independent pathways (Figure 2A).

Endothelial cells were initially considered not to respond to recombinant Shh directly, but rather to rely on adjacent mesenchymal cells thought to translate the presence of Hh ligand in signaling molecules that act on the endothelium (66). Particularly, activation of Shh signaling in intersticial mesenchymal cells enhances PTC, VEGF (Vascular Endothelial Growth Factor), and angiopoietin1 and -2 protein expressions (66). Further studies demonstrate that endothelial cells do respond to $\mathrm{Hh}$ inducing pro-angiogenic responses $(69,70)$. PI3K/Akt and SMO dependencies have been demonstrated to be essential for Shh signaling $(71,72)$. In agreement, Shh acutely stimulates fibroblast migration and endothelial cell tubulogenesis through Rho GTPases Racl and RhoA activation depending on SMO-G(i)-coupling and PI3K, but independently of Gli transcription factors $(69,73)$. Similarly, Shh promotes capillary morphogenesis and endothelial cell migration via a RhoA/ROCK dependent pathway, ultimately leading to increased expression of metalloproteinase 9 (MMP-9) or osteopontin downstream targets (74). Despite Gli nucleus translocation following Shh treatment of endothelial cells, role of these transcription factors in Shhangiogenesis responses is not fully understood (71). Controversy regarding Shh signaling is also present in vivo since Shh-induced angiogenesis is not affected in Gli1 null-mice (74), whereas capillaries morphogenesis is inhibited by the presence of Gli1 inhibitor GANT61 (70). Impaired angiogenesis in ischemic tissue of middleaged or old mice is associated with a decrease in Glil transcription factor mRNA expression $(68,75)$. These results reflect the complex interplay between canonical and non-canonical Shh pathways, or at least that of the Gli family of transcription factors, in the regulation of tubulogenesis and angiogenesis processes.

\section{EVs HARBORING Shh AS PROMISING THERAPEUTICAL DEVICES FOR CORRECTING ANGIOGENIC DEFECTS}

Shh beneficial effects in angiogenesis reveal therapeutic potential for stimulating neovascularization in disease states associated with impaired angiogenesis. This has led our group to engineer EVs specifically enriched in this morphogen and derived from activated T-lymphocytic cells. EVs ${ }^{\mathrm{Shh}+}$ are specifically retrieved from conditioned medium when lymphocytes are treated with a proactive stimulation, phytohemagglutin, and phorbol ester, followed by a pro-apoptotic treatment with actinomycin D (62). These $\mathrm{EVS}^{\mathrm{Shh}+}$ are functionally active and induce differentiation of megakaryocytes. EVs ${ }^{\mathrm{Shh}+}$ downstream effects likely depend on SMO activation, probably acting through direct Shh-PTC interaction (see Figure 2B). In vitro capillary formation by umbilical vein endothelial cells is both dependent on $\mathrm{EVs}^{\mathrm{Shh}}$concentrationand inhibited in presence of cyclopamine, which stabilizes SMO in an inactive form (76). However, whether $\mathrm{EVs}^{\mathrm{Shh}+}$ induce activation of Glil remain to be determined. In vitro, $\mathrm{EVS}^{\mathrm{Shh}+}$ increase mRNA levels of several pro-angiogenic factors, such as VEGF, angiopoietins, hepatocyte growth factor (HGF), interleukin-1 $\beta$, MMP-1 and decrease anti-angiogenic factors such as transforming growth factor $\beta$ (TGF $\beta$ ) (76). EVs ${ }^{\text {Shh }+}$ also increase cell adhesion and induce formation of stress fibers of in vitro endothelial cells in a SMO and Rho kinase pathways-dependent manner (76), likely signaling through the same pathway as described for recombinant Shh (69).

Angiogenic properties of $\mathrm{EVs}^{\mathrm{Shh}}+$ have been illustrated following their in vivo intravenous injection in mouse models (77). In vivo injection of $\mathrm{EVS} \mathrm{Shh}^{+}$in mice is able to improve endothelial function by increasing nitric oxide (NO) release and reversing endothelial dysfunction after ischemia/reperfusion (78). Indeed, $\mathrm{EVS} \mathrm{Sh}+$ trigger changes in the expression and phosphorylation of enzymes related to NO pathway, which seem to be dependent of the activation of kinases such as PI3K and ERK. Furthermore, 21 day-treatment of a model of mouse hind limb ischemia 


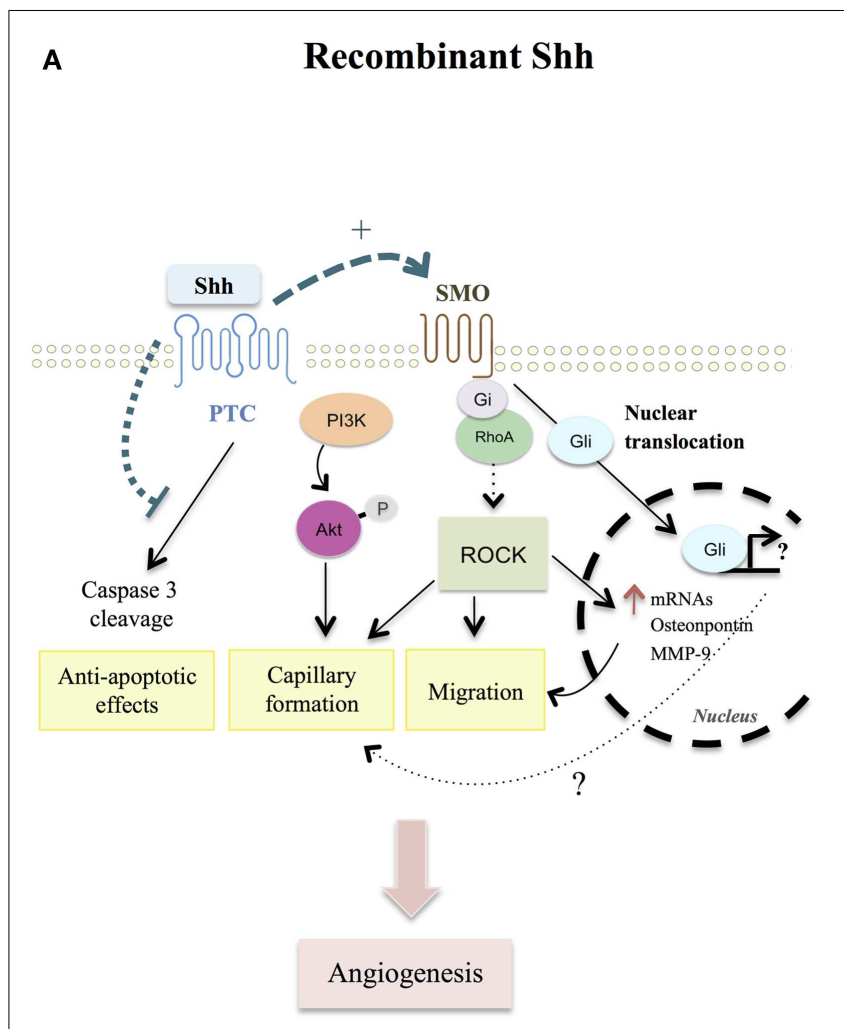

FIGURE 2 | Comparison between recombinant Shh- and $\mathrm{EVs}^{\mathrm{Shh}}$-activated pathways modulating angiogenesis and apoptosis in endothelial cells. (A) Recombinant Shh protein was shown to promote migration and capillary formation through SMO-Gi coupled and PI3K activation, triggering ROCK pathway activation, and downstream targets (MMP-9 and osteopontin). Such activation illustrates the importance of non-canonical pathways in Shh-induced angiogenesis processes. Despite Shh induction of Gli nuclear translocation in endothelial cells, role of canonical signaling on vascular function remains elusive. Finally, activation of $\mathrm{Hh}$ signaling counteracts non-canonical PTC-induced caspase-3 cleavage and protects endothelial cells against apoptosis. (B) $\mathrm{EVS}^{\mathrm{Shh}+}$ were demonstrated

\section{B EVs harboring Shh $\left(\mathrm{Evs}^{\mathrm{Shh}+}\right)$}

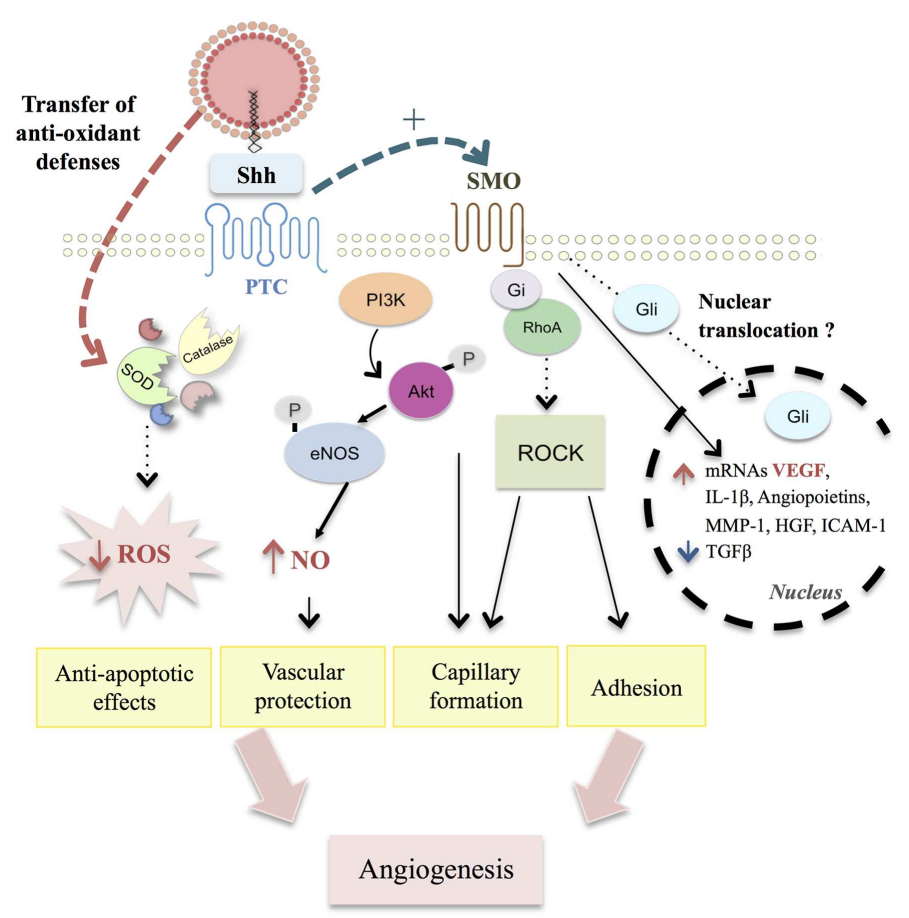

to use similar pathways than those described for recombinant Shh protein. They additionally induce functional and mature blood vessel formation by enhancing mRNA expression of several pro-angiogenic factors, especially VEGF and decreasing mRNA of anti-angiogenic TGF $\beta$. EVs ${ }^{\text {shh }}+$ also exert vasculoprotective effects by inducing anti-oxidants defenses, particularly through direct transfer of antioxidant enzymes (SOD, catalase) concurring to decrease ROS intracellular levels. SMO, smoothened; PI3K, phosphoinositide-3-kinase; ROCK, Rho-associated protein kinase; MMP-9, matrix metalloproteinase-9; Hh, Hedgehog; PTC, Patched; VEGF, vascular endothelial growth factor; TGF $\beta$, transforming growth factor $\beta$; SOD, superoxide dismutase; ROS, reactive oxygen species. with $\mathrm{EVS}$ Shh+ increase eNOS activation both in aorta and muscle as well as several pro-angiogenic factors (77). Taken together, lymphocytic-derived $\mathrm{EVs} \mathrm{Shh}$ are able to induce functional and mature blood vessel formation by reciprocally regulating the proand anti-angiogenic factors.

Apart from directly stimulating angiogenesis processes, $\mathrm{EVs}^{\mathrm{Shh}+}$ reveal anti-apoptotic properties notably by decreasing reactive oxygen species (ROS) production (78). Mechanistically, $\mathrm{EVs}^{\mathrm{Shh}+}$ exert their vasculoprotective effects by promoting internalization and induction of antioxidant messages to the endothelial monolayer. Indeed, $\mathrm{EVs}^{\mathrm{Shh}+}$ prevent apoptosis in endothelial cells induced by actinomycin D by transferring functional antioxidant enzymes [superoxide dismutase (SOD) isoforms and catalase] and by inducing cellular Mn-SOD protein expression (79). Anti-apoptotic effects have also been reported following Hh ligand treatment of endothelial cells, but rather relying on the inhibition of the pro-apoptotic effect of PTC, normally leading to caspase-3 cleavage (69).
Therefore, EVs ${ }^{\mathrm{Shh}+}$ may represent a powerful tool in diseases associated with failed angiogenesis, associated with their proangiogenic and anti-apoptotic properties. In addition to observed differences in regulated pathways, structural features of Shh associated with EVs might be of particular importance. EVs provide a lipidic environment analogous to cell membrane, which could influence Shh downstream signaling considering the importance of these lipid-adducts in Shh activity $(57,58)$. Finally, EV-delivery of Shh protein seems to represent a major mechanism in the observed preservation of cardiac function in stem cell-based therapies. Indeed, $\mathrm{CD}^{+}{ }^{+}$cells genetically modified to express Shh $\left[\mathrm{CD} 34^{+}(\mathrm{Shh})\right]$ offer a better protection against ventricular dilation and cardiac functional decline than $\mathrm{CD} 34^{+}$cells alone when injected into mice after acute myocardial infarction (80). In vitro analysis of exosomes derived from $\mathrm{CD}_{3} 4^{+}(\mathrm{Shh})$ further revealed the functional transfer and activation of Shh signaling in recipient cells, which might explain the observed preservation of cardiac function in mice treated with $\mathrm{CD}^{+} 4^{+}$(Shh) EVs. 
Thus, EVs harboring Shh represent an exciting tool for therapy regarding angiogenesis defects related diseases and cardioprotection.

\section{TOWARD CLINICAL TRANSLATION}

Extracellular vesicles might be further used as delivery devices for any molecule of interest. In particular, using naturally occurring secreted vesicles might allow overcoming toxicity or immunogenicity associated with other developed carrying agents like liposomes or nanoparticles. Moreover, this new drug-delivery system might be combined with other existing therapeutic strategies to optimize drug-delivery. Indeed, a recent report demonstrates that adeno-associated viruses (AAVs) are more efficient when encapsulated in EVs (termed vexosomes) than free AAVs for the delivery of cargo into recipient cells. Such a unique entity offers a promising strategy to improve gene delivery (81). Alternatively, tumor cells incubated with chemotherapeutic drugs are able to package these drugs into EVs, which can be collected and used to effectively kill tumor cells in murine tumor models without typical side effects (82).

Furthermore, the use of biological material as carriers might also allow getting molecules in their native conformation, due to natural interaction or folding with membranous environment. Different strategies might be used to specifically target molecules in EVs. For example, addition of different types of membrane anchors can target highly oligomeric cytoplasmic proteins to sites of vesicle secretion and EVs (83). Another important issue is the biodistribution of EVs. Indeed, EVs are circulating through many fluids, and have been especially recovered from blood or lymph, therefore allowing their large dissemination in the organism. Such circulating properties are of particular interest in the treatment of cardiovascular alterations since the entire vascular network would be exposed to EVs. Since dissemination or accumulation of these biological vectors may also be hindered, one could need to specifically target cargo delivery to enhance EV properties. Elegant studies using engineering donor cells expressing peptide targeting-ligand subsequently recovered in EVs produced by cells have respectively allowed to specifically target neurons (84) and breast cancer cells (85).

Despite interesting perspectives for cardiovascular treatment, EV-based therapies still need more investigations to translate into clinical studies. Lessons from the ongoing exosome-based preliminary clinical trials in the field of cancer would certainly boost such therapeutic perspectives. Particularly, one challenge would be to control the fragile balance between the harmful effects and beneficial ones reported for EVs in the context of cardiovascular diseases. However, cardiovascular field would undoubtedly benefit from optimized developed methods for large-scale production of clinical grade EVs derived from MSC (27) or dendritic cells (86).

By inhibiting their deleterious effects, by taking advantage of their regenerative properties or by developing EV-based drugdelivery tools, targeting EVs reveal all the exciting opportunities to treat cardiovascular alterations by non-immunogenic means. In particular, EVs carrying $\mathrm{Shh}^{+}$represent interesting tools to treat diseases related to failed NO pathway and impaired angiogenesis.

\section{ACKNOWLEDGMENTS}

Soazig Le Lay is funded by a grant from ANR, MilkChEST n ${ }^{\circ}$ ANR12-BSV6-0013-04 and by a grant from "Région Pays de la Loire". Audrey Fleury received a Ph.D fellowship from "Région Pays de la Loire".

\section{REFERENCES}

1. van der Pol E, Boing AN, Harrison P, Sturk A, Nieuwland R. Classification, functions, and clinical relevance of extracellular vesicles. Pharmacol Rev (2012) 64:676-705. doi:10.1124/pr.112.005983

2. Witwer KW, Buzas EI, Bemis LT, Bora A, Lasser C, Lotvall J, et al. Standardization of sample collection, isolation and analysis methods in extracellular vesicle research. J Extracell Vesicles (2013) 2:20360. doi:10.3402/jev.v2i0.20360

3. Gaceb A, Martinez MC, Andriantsitohaina R. Extracellular vesicles: new players in cardiovascular diseases. Int J Biochem Cell Biol (2014) 50:24-8. doi:10.1016/ j.biocel.2014.01.018

4. Schiro A, Wilkinson FL, Weston R, Smyth JV, Serracino-Inglott F, Alexander MY. Endothelial microparticles as conveyors of information in atherosclerotic disease. Atherosclerosis (2014) 234:295-302. doi:10.1016/j.atherosclerosis.2014. 03.019

5. Amabile N, Cheng S, Renard JM, Larson MG, Ghorbani A, Mccabe E, et al. Association of circulating endothelial microparticles with cardiometabolic risk factors in the Framingham Heart Study. Eur Heart J (2014). doi:10.1093/eurheartj/ ehu153

6. Martinez MC, Tual-Chalot S, Leonetti D, Andriantsitohaina R. Microparticles: targets and tools in cardiovascular disease. Trends Pharmacol Sci (2011) 32:659-65. doi:10.1016/j.tips.2011.06.005

7. El Andaloussi S, Mager I, Breakefield XO, Wood MJ. Extracellular vesicles: biology and emerging therapeutic opportunities. Nat Rev Drug Discov (2013) 12:347-57. doi:10.1038/nrd3978

8. Chalmin F, Ladoire S, Mignot G, Vincent J, Bruchard M, Remy-Martin JP, et al. Membrane-associated Hsp72 from tumor-derived exosomes mediates STAT3-dependent immunosuppressive function of mouse and human myeloid-derived suppressor cells. J Clin Invest (2010) 120:457-71. doi:10.1172/ JCI40483

9. Loyer X, Vion AC, Tedgui A, Boulanger CM. Microvesicles as cell-cell messengers in cardiovascular diseases. Circ Res (2014) 114:345-53. doi:10.1161/ CIRCRESAHA.113.300858

10. Smalheiser NR. Do neural cells communicate with endothelial cells via secretory exosomes and microvesicles? Cardiovasc Psychiatry Neurol (2009) 2009:383086. doi: $10.1155 / 2009 / 383086$

11. Mostefai HA, Agouni A, Carusio N, Mastronardi ML, Heymes C, Henrion D, et al. Phosphatidylinositol 3-kinase and xanthine oxidase regulate nitric oxide and reactive oxygen species productions by apoptotic lymphocyte microparticles in endothelial cells. J Immunol (2008) 180:5028-35. doi:10.4049/jimmunol. 180.7.5028

12. Soriano AO, Jy W, Chirinos JA, Valdivia MA, Velasquez HS, Jimenez JJ, et al. Levels of endothelial and platelet microparticles and their interactions with leukocytes negatively correlate with organ dysfunction and predict mortality in severe sepsis. Crit Care Med (2005) 33:2540-6. doi:10.1097/01.CCM. 0000186414.86162 .03

13. Mangi AA, Noiseux N, Kong D, He H, Rezvani M, Ingwall JS, et al. Mesenchymal stem cells modified with Akt prevent remodeling and restore performance of infarcted hearts. Nat Med (2003) 9:1195-201. doi:10.1038/nm912

14. Gnecchi M, Danieli P, Cervio E. Mesenchymal stem cell therapy for heart disease. Vascul Pharmacol (2012) 57:48-55. doi:10.1016/j.vph.2012.04.002

15. Ratajczak MZ, Kucia M, Jadczyk T, Greco NJ, Wojakowski W, Tendera M, et al. Pivotal role of paracrine effects in stem cell therapies in regenerative medicine: can we translate stem cell-secreted paracrine factors and microvesicles into better therapeutic strategies? Leukemia (2012) 26:1166-73. doi:10.1038/ leu.2011.389

16. Gnecchi M, He H, Liang OD, Melo LG, Morello F, Mu H, et al. Paracrine action accounts for marked protection of ischemic heart by Akt-modified mesenchymal stem cells. Nat Med (2005) 11:367-8. doi:10.1038/nm0405-367

17. Gnecchi M, He H, Noiseux N, Liang OD, Zhang L, Morello F, et al. Evidence supporting paracrine hypothesis for Akt-modified mesenchymal stem cell-mediated cardiac protection and functional improvement. FASEB J (2006) 20:661-9. doi:10.1096/fj.05-5211com 
18. Timmers L, Lim SK, Arslan F, Armstrong JS, Hoefer IE, Doevendans PA, et al. Reduction of myocardial infarct size by human mesenchymal stem cell conditioned medium. Stem Cell Res (2007) 1:129-37. doi:10.1016/j.scr.2008.02.002

19. Lai RC, Arslan F, Lee MM, Sze NS, Choo A, Chen TS, et al. Exosome secreted by MSC reduces myocardial ischemia/reperfusion injury. Stem Cell Res (2010) 4:214-22. doi:10.1016/j.scr.2009.12.003

20. Arslan F, Lai RC, Smeets MB, Akeroyd L, Choo A, Aguor EN, et al. Mesenchymal stem cell-derived exosomes increase ATP levels, decrease oxidative stress and activate PI3K/Akt pathway to enhance myocardial viability and prevent adverse remodeling after myocardial ischemia/reperfusion injury. Stem Cell Res (2013) 10:301-12. doi:10.1016/j.scr.2013.01.002

21. Sahoo S, Klychko E, Thorne T, Misener S, Schultz KM, Millay M, et al. Exosomes from human CD34(+) stem cells mediate their proangiogenic paracrine activity. Circ Res (2011) 109:724-8. doi:10.1161/CIRCRESAHA.111.253286

22. Cantaluppi V, Biancone L, Figliolini F, Beltramo S, Medica D, Deregibus MC, et al. Microvesicles derived from endothelial progenitor cells enhance neoangiogenesis of human pancreatic islets. Cell Transplant (2012) 21:1305-20. doi:10.3727/096368911X627534

23. Vrijsen KR, Sluijter JP, Schuchardt MW, Van Balkom BW, Noort WA, Chamuleau SA, et al. Cardiomyocyte progenitor cell-derived exosomes stimulate migration of endothelial cells. J Cell Mol Med (2010) 14:1064-70. doi:10.1111/j.1582-4934. 2010.01081.x

24. Chen L, Wang Y, Pan Y, Zhang L, Shen C, Qin G, et al. Cardiac progenitor-derived exosomes protect ischemic myocardium from acute ischemia/reperfusion injury. Biochem Biophys Res Commun (2013) 431:566-71. doi:10.1016/j.bbrc.2013.01. 015

25. Barile L, Gherghiceanu M, Popescu LM, Moccetti T, Vassalli G. Ultrastructural evidence of exosome secretion by progenitor cells in adult mouse myocardium and adult human cardiospheres. J Biomed Biotechnol (2012) 2012:354605. doi:10.1155/2012/354605

26. Chaput N, Thery C. Exosomes: immune properties and potential clinical implementations. Semin Immunopathol (2011) 33:419-40. doi:10.1007/s00281-0100233-9

27. Chen TS, Arslan F, Yin Y, Tan SS, Lai RC, Choo AB, et al. Enabling a robust scalable manufacturing process for therapeutic exosomes through oncogenic immortalization of human ESC-derived MSCs. J Transl Med (2011) 9:47. doi:10.1186/1479-5876-9-47

28. Fonsato V, Collino F, Herrera MB, Cavallari C, Deregibus MC, Cisterna B, et al. Human liver stem cell-derived microvesicles inhibit hepatoma growth in SCID mice by delivering antitumor microRNAs. Stem Cells (2012) 30:1985-98. doi: $10.1002 /$ stem. 1161

29. Zhu W, Huang L, Li Y, Zhang X, Gu J, Yan Y, et al. Exosomes derived from human bone marrow mesenchymal stem cells promote tumor growth in vivo. Cancer Lett (2012) 315:28-37. doi:10.1016/j.canlet.2011.10.002

30. Ratajczak J, Miekus K, Kucia M, Zhang J, Reca R, Dvorak P, et al. Embryonic stem cell-derived microvesicles reprogram hematopoietic progenitors: evidence for horizontal transfer of mRNA and protein delivery. Leukemia (2006) 20:847-56. doi:10.1038/sj.leu.2404132

31. Deregibus MC, Cantaluppi V, Calogero R, Lo Iacono M, Tetta C, Biancone L, et al. Endothelial progenitor cell derived microvesicles activate an angiogenic program in endothelial cells by a horizontal transfer of mRNA. Blood (2007) 110:2440-8. doi:10.1182/blood-2007-03-078709

32. Valadi H, Ekstrom K, Bossios A, Sjostrand M, Lee JJ, Lotvall JO. Exosomemediated transfer of mRNAs and microRNAs is a novel mechanism of genetic exchange between cells. Nat Cell Biol (2007) 9:654-9. doi:10.1038/ncb1596

33. Skog J, Wurdinger T, Van Rijn S, Meijer DH, Gainche L, Sena-Esteves M, et al. Glioblastoma microvesicles transport RNA and proteins that promote tumour growth and provide diagnostic biomarkers. Nat Cell Biol (2008) 10:1470-6. doi:10.1038/ncb1800

34. Aliotta JM, Pereira M, Johnson KW, De Paz N, Dooner MS, Puente N, et al. Microvesicle entry into marrow cells mediates tissue-specific changes in mRNA by direct delivery of mRNA and induction of transcription. Exp Hematol (2010) 38:233-45. doi:10.1016/j.exphem.2010.01.002

35. Collino F, Deregibus MC, Bruno S, Sterpone L, Aghemo G, Viltono L, et al. Microvesicles derived from adult human bone marrow and tissue specific mesenchymal stem cells shuttle selected pattern of miRNAs. PLoS One (2010) 5:e11803. doi:10.1371/journal.pone. 0011803
36. Mittelbrunn M, Gutierrez-Vazquez C, Villarroya-Beltri C, Gonzalez S, SanchezCabo F, Gonzalez MA, et al. Unidirectional transfer of microRNA-loaded exosomes from T cells to antigen-presenting cells. Nat Commun (2011) 2:282. doi:10.1038/ncomms1285

37. Montecalvo A, Larregina AT, Shufesky WJ, Stolz DB, Sullivan ML, Karlsson JM, et al. Mechanism of transfer of functional microRNAs between mouse dendritic cells via exosomes. Blood (2012) 119:756-66. doi:10.1182/blood-201102-338004

38. Ikeda S, Kong SW, Lu J, Bisping E, Zhang H, Allen PD, et al. Altered microRNA expression in human heart disease. Physiol Genomics (2007) 31:367-73. doi:10. 1152/physiolgenomics.00144.2007

39. Zampetaki A, Mayr M. MicroRNAs in vascular and metabolic disease. Circ Res (2012) 110:508-22. doi:10.1161/CIRCRESAHA.111.247445

40. Diehl P, Fricke A, Sander L, Stamm J, Bassler N, Htun N, et al. Microparticles: major transport vehicles for distinct microRNAs in circulation. Cardiovasc Res (2012) 93:633-44. doi:10.1093/cvr/cvs007

41. Zhang Y, Liu D, Chen X, Li J, Li L, Bian Z, et al. Secreted monocytic miR150 enhances targeted endothelial cell migration. Mol Cell (2010) 39:133-44. doi:10.1016/j.molcel.2010.06.010

42. Villarroya-Beltri C, Gutierrez-Vazquez C, Sanchez-Cabo F, Perez-Hernandez D, Vazquez J, Martin-Cofreces N, et al. Sumoylated hnRNPA2B1 controls the sorting of miRNAs into exosomes through binding to specific motifs. Nat Commun (2013) 4:2980. doi:10.1038/ncomms3980

43. Cantaluppi V, Gatti S, Medica D, Figliolini F, Bruno S, Deregibus MC, et al. Microvesicles derived from endothelial progenitor cells protect the kidney from ischemia-reperfusion injury by microRNA-dependent reprogramming of resident renal cells. Kidney Int (2012) 82:412-27. doi:10.1038/ki.2012.105

44. Akao Y, Iio A, Itoh T, Noguchi S, Itoh Y, Ohtsuki Y, et al. Microvesicle-mediated RNA molecule delivery system using monocytes/macrophages. Mol Ther (2011) 19:395-9. doi:10.1038/mt.2010.254

45. Fish JE, Santoro MM, Morton SU, Yu S, Yeh RF, Wythe JD, et al. miR-126 regulates angiogenic signaling and vascular integrity. Dev Cell (2008) 15:272-84 doi:10.1016/j.devcel.2008.07.008

46. Wang S, Aurora AB, Johnson BA, Qi X, Mcanally J, Hill JA, et al. The endothelialspecific microRNA miR-126 governs vascular integrity and angiogenesis. Dev Cell (2008) 15:261-71. doi:10.1016/j.devcel.2008.07.002

47. Zernecke A, Bidzhekov K, Noels H, Shagdarsuren E, Gan L, Denecke B, et al. Delivery of microRNA-126 by apoptotic bodies induces CXCL12-dependent vascular protection. Sci Signal (2009) 2:ra81. doi:10.1126/scisignal.2000610

48. Jansen F, Yang X, Hoelscher M, Cattelan A, Schmitz T, Proebsting S, et al. Endothelial microparticle-mediated transfer of MicroRNA-126 promotes vascular endothelial cell repair via SPRED1 and is abrogated in glucosedamaged endothelial microparticles. Circulation (2013) 128:2026-38. doi:10. 1161/CIRCULATIONAHA.113.001720

49. Ranghino A, Cantaluppi V, Grange C, Vitillo L, Fop F, Biancone L, et al. Endothelial progenitor cell-derived microvesicles improve neovascularization in a murine model of hindlimb ischemia. Int J Immunopathol Pharmacol (2012) 25:75-85.

50. Fichtlscherer S, De Rosa S, Fox H, Schwietz T, Fischer A, Liebetrau C, et al. Circulating microRNAs in patients with coronary artery disease. Circ Res (2010) 107:677-84. doi:10.1161/CIRCRESAHA.109.215566

51. Chiang C, Litingtung Y, Lee E, Young KE, Corden JL, Westphal H, et al. Cyclopia and defective axial patterning in mice lacking sonic hedgehog gene function. Nature (1996) 383:407-13. doi:10.1038/383407a0

52. Ingham PW, McMahon AP. Hedgehog signaling in animal development: paradigms and principles. Genes Dev (2001) 15:3059-87. doi:10.1101/gad.938601

53. Porter JA, Ekker SC, Park WJ, Von Kessler DP, Young KE, Chen CH, et al. Hedgehog patterning activity: role of a lipophilic modification mediated by the carboxy-terminal autoprocessing domain. Cell (1996) 86:21-34. doi:10.1016/ S0092-8674(00)80074-4

54. Chen X, Tukachinsky H, Huang CH, Jao C, Chu YR, Tang HY, et al. Processing and turnover of the hedgehog protein in the endoplasmic reticulum. J Cell Biol (2011) 192:825-38. doi:10.1083/jcb.201008090

55. Chamoun Z, Mann RK, Nellen D, Von Kessler DP, Bellotto M, Beachy PA, et al. Skinny hedgehog, an acyltransferase required for palmitoylation and activity of the hedgehog signal. Science (2001) 293:2080-4. doi:10.1126/science. 1064437 
56. Cooper MK, Wassif CA, Krakowiak PA, Taipale J, Gong R, Kelley RI, et al. A defective response to hedgehog signaling in disorders of cholesterol biosynthesis. Nat Genet (2003) 33:508-13. doi:10.1038/ng1134

57. Lewis PM, Dunn MP, Mcmahon JA, Logan M, Martin JF, St-Jacques B, et al. Cholesterol modification of sonic hedgehog is required for long-range signaling activity and effective modulation of signaling by Ptc1. Cell (2001) 105:599-612. doi:10.1016/S0092-8674(01)00369-5

58. Chen MH, Li YJ, Kawakami T, Xu SM, Chuang PT. Palmitoylation is required for the production of a soluble multimeric hedgehog protein complex and longrange signaling in vertebrates. Genes Dev (2004) 18:641-59. doi:10.1101/gad. 1185804

59. Briscoe J, Therond PP. The mechanisms of hedgehog signalling and its roles in development and disease. Nat Rev Mol Cell Biol (2013) 14:416-29. doi:10.1038/ nrm3598

60. Tanaka Y, Okada Y, Hirokawa N. FGF-induced vesicular release of sonic hedgehog and retinoic acid in leftward nodal flow is critical for left-right determination. Nature (2005) 435:172-7. doi:10.1038/nature03494

61. Liegeois S, Benedetto A, Garnier JM, Schwab Y, Labouesse M. The V0-ATPase mediates apical secretion of exosomes containing hedgehog-related proteins in Caenorhabditis elegans. J Cell Biol (2006) 173:949-61. doi:10.1083/jcb. 200511072

62. Martinez MC, Larbret F, Zobairi F, Coulombe J, Debili N, Vainchenker W, et al. Transfer of differentiation signal by membrane microvesicles harboring hedgehog morphogens. Blood (2006) 108:3012-20. doi:10.1182/blood-200604-019109

63. Ruat M, Hoch L, Faure H, Rognan D. Targeting of smoothened for therapeutic gain. Trends Pharmacol Sci (2014) 35:237-46. doi:10.1016/j.tips.2014.03.002

64. Teperino R, Aberger F, Esterbauer H, Riobo N, Pospisilik JA. Canonical and non-canonical hedgehog signalling and the control of metabolism. Semin Cell Dev Biol (2014). doi:10.1016/j.semcdb.2014.05.007

65. Soleti R, Martinez MC. Sonic hedgehog on microparticles and neovascularization. Vitam Horm (2012) 88:395-438. doi:10.1016/B978-0-12-394622-5. 00018-3

66. Pola R, Ling LE, Silver M, Corbley MJ, Kearney M, Blake Pepinsky R, et al. The morphogen sonic hedgehog is an indirect angiogenic agent upregulating two families of angiogenic growth factors. Nat Med (2001) 7:706-11. doi:10.1038/89083

67. Kusano KF, Pola R, Murayama T, Curry C, Kawamoto A, Iwakura A, et al. Sonic hedgehog myocardial gene therapy: tissue repair through transient reconstitution of embryonic signaling. Nat Med (2005) 11:1197-204. doi:10.1038/nm1313

68. Palladino M, Gatto I, Neri V, Straino S, Silver M, Tritarelli A, et al. Pleiotropic beneficial effects of sonic hedgehog gene therapy in an experimental model of peripheral limb ischemia. Mol Ther (2011) 19:658-66. doi:10.1038/mt.2010.292

69. Chinchilla P, Xiao L, Kazanietz MG, Riobo NA. Hedgehog proteins activate pro-angiogenic responses in endothelial cells through non-canonical signaling pathways. Cell Cycle (2010) 9:570-9. doi:10.4161/cc.9.3.10591

70. Spek CA, Bijlsma MF, Queiroz KC. Canonical hedgehog signaling drives proangiogenic responses in endothelial cells. Cell Cycle (2010) 9:1683. doi:10.4161/cc. 9.9.11653

71. Kanda S, Mochizuki Y, Suematsu T, Miyata Y, Nomata K, Kanetake H. Sonic hedgehog induces capillary morphogenesis by endothelial cells through phosphoinositide 3-kinase. J Biol Chem (2003) 278:8244-9. doi:10.1074/jbc. M210635200

72. Riobo NA, Lu K, Ai X, Haines GM, Emerson CP Jr. Phosphoinositide 3-kinase and Akt are essential for sonic hedgehog signaling. Proc Natl Acad Sci U S A (2006) 103:4505-10. doi:10.1073/pnas.0504337103

73. Polizio AH, Chinchilla P, Chen X, Kim S, Manning DR, Riobo NA. Heterotrimeric Gi proteins link hedgehog signaling to activation of Rho small GTPases to promote fibroblast migration. J Biol Chem (2011) 286:19589-96. doi:10.1074/jbc.M110.197111
74. Renault MA, Roncalli J, Tongers J, Thorne T, Klyachko E, Misener S, et al. Sonic hedgehog induces angiogenesis via Rho kinase-dependent signaling in endothelial cells. J Mol Cell Cardiol (2010) 49:490-8. doi:10.1016/j.yjmcc.2010.05.003

75. Renault MA, Robbesyn F, Chapouly C, Yao Q, Vandierdonck S, Reynaud A, et al. Hedgehog-dependent regulation of angiogenesis and myogenesis is impaired in aged mice. Arterioscler Thromb Vasc Biol (2013) 33:2858-66. doi:10.1161/ATVBAHA.113.302494

76. Soleti R, Benameur T, Porro C, Panaro MA, Andriantsitohaina R, Martinez MC. Microparticles harboring sonic hedgehog promote angiogenesis through the upregulation of adhesion proteins and proangiogenic factors. Carcinogenesis (2009) 30:580-8. doi:10.1093/carcin/bgp030

77. Benameur T, Soleti R, Porro C, Andriantsitohaina R, Martinez MC. Microparticles carrying sonic hedgehog favor neovascularization through the activation of nitric oxide pathway in mice. PLoS One (2010) 5:e12688. doi:10.1371/journal. pone. 0012688

78. Agouni A, Mostefai HA, Porro C, Carusio N, Favre J, Richard V, et al. Sonic hedgehog carried by microparticles corrects endothelial injury through nitric oxide release. FASEB J (2007) 21:2735-41. doi:10.1096/fj.07-8079com

79. Soleti R, Lauret E, Andriantsitohaina R, Carmen Martinez M. Internalization and induction of antioxidant messages by microvesicles contribute to the antiapoptotic effects on human endothelial cells. Free Radic Biol Med (2012) 53:2159-70. doi:10.1016/j.freeradbiomed.2012.09.021

80. Mackie AR, Klyachko E, Thorne T, Schultz KM, Millay M, Ito A, et al. Sonic hedgehog-modified human CD34+ cells preserve cardiac function after acute myocardial infarction. Circ Res (2012) 111:312-21. doi:10.1161/CIRCRESAHA. 112.266015

81. Maguire CA, Balaj L, Sivaraman S, Crommentuijn MH, Ericsson M, MinchevaNilsson L, et al. Microvesicle-associated AAV vector as a novel gene delivery system. Mol Ther (2012) 20:960-71. doi:10.1038/mt.2011.303

82. Tang K, Zhang Y, Zhang H, Xu P, Liu J, Ma J, et al. Delivery of chemotherapeutic drugs in tumour cell-derived microparticles. Nat Commun (2012) 3:1282. doi:10.1038/ncomms2282

83. Shen B, Wu N, Yang JM, Gould SJ. Protein targeting to exosomes/microvesicles by plasma membrane anchors. J Biol Chem (2011) 286:14383-95. doi:10.1074/ jbc.M110.208660

84. Alvarez-Erviti L, Seow Y, Yin H, Betts C, Lakhal S, Wood MJ. Delivery of siRNA to the mouse brain by systemic injection of targeted exosomes. Nat Biotechnol (2011) 29:341-5. doi:10.1038/nbt.1807

85. Ohno S, Takanashi M, Sudo K, Ueda S, Ishikawa A, Matsuyama N, et al. Systemically injected exosomes targeted to EGFR deliver antitumor microRNA to breast cancer cells. Mol Ther (2013) 21:185-91. doi:10.1038/mt.2012.180

86. Yeo RW, Lai RC, Zhang B, Tan SS, Yin Y, Teh BJ, et al. Mesenchymal stem cell: an efficient mass producer of exosomes for drug delivery. Adv Drug Deliv Rev (2013) 65:336-41. doi:10.1016/j.addr.2012.07.001

Conflict of Interest Statement: The authors declare that the research was conducted in the absence of any commercial or financial relationships that could be construed as a potential conflict of interest.

Received: 24 June 2014; accepted: 18 July 2014; published online: 04 August 2014. Citation: Fleury A, Martinez MC and Le Lay S (2014) Extracellular vesicles as therapeutic tools in cardiovascular diseases. Front. Immunol. 5:370. doi: 10.3389/fimmu.2014.00370

This article was submitted to Immunotherapies and Vaccines, a section of the journal Frontiers in Immunology.

Copyright (C) 2014 Fleury, Martinez and Le Lay. This is an open-access article distributed under the terms of the Creative Commons Attribution License (CC BY). The use, distribution or reproduction in other forums is permitted, provided the original author(s) or licensor are credited and that the original publication in this journal is cited, in accordance with accepted academic practice. No use, distribution or reproduction is permitted which does not comply with these terms. 